\title{
Determining design requirements for active aging: Personas, experience maps, and stakeholders
}

\author{
Barbara Schäpers ${ }^{\mathrm{a}, *}$ \\ Carmen Krewer PhD \\ Mika Yasuoka $\mathrm{PhD}^{\mathrm{b}}$ \\ Daniela Becker ${ }^{\mathrm{a}}$ \\ Silke Heller MSc ${ }^{\mathrm{a}}$ \\ Marcus Kaiser ${ }^{a}$ \\ Eberhard Koenig MD PhDa \\ Friedemann Müller MD MSc PhD
}

\begin{abstract}
${ }^{a}$ Schön Klinik Bad Aibling SE \& Co. KG, Science Department, Bad Aibling, Germany; ${ }^{\text {bTechni- }}$ cal University of Denmark, Management Engineering, Lyngby, Denmark; *Corresponding author: bschaepers@schoen-kliniken.de
\end{abstract}

\begin{abstract}
B. Schäpers, C. Krewer, M. Yasuoka, D. Becker, S. Heller, M. Kaiser, E. Koenig, F. Müller. Determining design requirements for active aging: Personas, experience maps, and stakeholders. Gerontechnology 2017;16(3):139-150; https://doi.org/10.4017/gt.2017.16.3.003.00 In this European multicenter project REACH-Responsive Engagement of the older adults promoting Activity and Customized Health care (REACH), a sensing-monitoring-intervention system is being developed that can be placed in an unobtrusive manner in various care settings and living environments of the older adults. In order to develop such a complex user-centered system, experts from several professions needed to collaborate in a joint development team. To have a successful development progress, it is essential to perform requirement specification and analysis in the beginning of the project. A description of the targeted end-user including the user environment and an analysis of the entities associated with the user and thus associated with the system is needed. Different methods are used to describe and analyze these important components of the REACH system, such as describing use cases, creating personas, developing experience maps, and defining and analyzing stakeholders. The methods used are described and the findings are reported.
\end{abstract}

Keywords: independent living; smart technology; health; older adults; active life

The REACH consortium is developing a sensingmonitoring-intervention system that can be placed in an unobtrusive manner in various care settings and living environments of the older adults. The system will be able to (1) use a set of sensors to detect selected vital signs, behavioral and care patterns, and health states; (2) predict as early as possible future health states, risks, or events such as the loss of function, frailty, stroke, etc.; and (3) provide and coordinate proactively a set of customized products and services with the overall aim of supporting and promoting physical activity, including related social activities and serious games. The system's main task is preventing or delaying functional loss and reinforcing the functional ability of the older adults to prolong independent living.
In order to develop these features in a target-oriented manner, REACH integrates knowledge providers (research entities and universities), technology providers (manufacturers of sensors, software, and intervention systems), and solution operators (hospitals, rehabilitation centers, care homes, and home care providers) into a joint development team, as well as multiplicators (insurance providers, standardization organizations, etc.).

The solution operators (use cases) manage different levels of complex dependency needs of the older adults ${ }^{1}$. The needs can originate from specific disease-based deficits or from general age-related degeneration. The use cases reflect specific application settings for the REACH sys- 
tem, such as an acute hospital setting covered by the Hôpitaux Universitaires des Genève (HUG) in Switzerland, a rehabilitation setting covered by the Schön Klinik Bad Aibling SE \& Co. KG (SK) in Germany, and the home care setting covered by the Lyngby-Taarbæk Kommune (LYNGBY) in Denmark, and Stichting Zuidzorg Extra (ZZ) in Netherlands. In addition, ZZ covers the care home setting. In the Dutch and Danish home care setting, seniors $65+$ tend to live alone or with their senior partners but not with their children or relatives ${ }^{2,3}$. LYNGBY and ZZ also offer support to the older adults living at home but who are in need of assistance with their activities of daily living (ADL; such as, personal hygiene, sufficient fluid intake, and household activities) ${ }^{4}$ and to improve their quality of life (such as social contacts and hobbies).

In order to develop the REACH system in a target-oriented manner, the setting in which the system will be applied in the future has to be well known. It is essential to know all aspects significant to the system beforehand to prevent misconceptions. The REACH system should be able to move with the older adults through various use cases, health states, and institutions and finally motivate and support them to an active and healthy life at home. These concepts allow a multidimensional visualization of the system setting and provide user characteristics as a common reference for the development teams.

The way stakeholders, personas, and experience mapping contribute to define the system requirements is described in the following:

Data from stakeholder analysis are necessary for the system developers to define interfaces and data flow charts and assign access rights. To design a marketable product, the financial structure of the system environment (interest and influence of stakeholders) in addition has to be taken into account.

Personas provide detailed information about the needs and expectations of the core user, the patients and older adults. The functionality of the system will be designed primarily to meet their requirements. Personas transfer information from the strategical to the technical developers by visualizing the user profile into a generally understandable format.

The experience mapping goes even further and allows deep insights into the personal experiences of the user. This kind of information enables the developers to generate individual applications, tasks, and feedback.

The use case partners represent REACH's 'system development strategy'. In the early phase of the system development, the focus will be on the structured settings (hospital and rehabilitation) for efficient requirement specification and analysis. Later the development focuses on the open settings that support the older adults at home in many different scenarios. The outcome of the analysis shows the situation before intervention based on the use case partner's settings and enables the modeling of REACH's use scenarios.

The 4 use cases, acute hospital, rehabilitation hospital (HUG and SK), care home, and home care (LYNGBY and ZZ) cover most scenarios where the REACH system could support a patient through the recovery process (Figure 1).

HUG with its acute and geriatric unit, and rehabilitation and home care specialists will focus on acute care, the transition between the use cases, and the health states of end-users. SK will focus on physical and cognitive rehabilitation. Rehabilitation aims at reducing the impairment and handicap of patients/older adults, thus reducing their need for care and support. The treatment is based on relearning lost abilities and is adapted to the patients' individual capabilities. During rehabilitation the patients use assistive devices and acquire compensation strategies to foster independent living. ZZ and LYNGBY are the use cases representing the environment of the older adults at home. To reduce the risk of health deterioration caused by the natural aging process, physical and cognitive training and sometimes therapy are needed. The natural aging process may be negatively affected by complications and adverse events that may cause accelerated deterioration and a decline in the health status ${ }^{4}$. REACH will prevent negative consequences in a variety of ways, for example, by increasing the activity level, supporting social interaction, or motivating the performance of cognitive training. ZZ will focus on home care, representing end-users with relatively good basic health in the care continuum of REACH. These persons receive some sort of care or household services and need to be motivated for physical and cognitive activity. Patients may receive in-home rehabilitation therapies addressing mild disabilities in order to avoid nursing home or hospital admission. LYNGBY will focus on home care and the older adults living in smart homes. In the REACH care continuum, the older adults supported by LYNGBY with relatively good health need to be motivated for physical and cognitive activity (including ADL training) ${ }^{4}$. Those with lighter disabilities need rehabilitation at home to avoid nursing home or hospital/acute care admission.

Some scenarios are overlapping and can be found in more than 1 use case. The modularity of the REACH system should allow customization with 
regard to varying needs of the older adults during their life journey. The use cases are not only separate settings in which REACH functionality can be integrated; they can also be brought into a logical arrangement that represents the care continuum and the transition between health states.

Based on a patient's condition, not all use cases have to be passed through by each patient and the duration of stay in the respective use case can vary significantly. Fast recovery or complications and other (adverse) events may alter the patient's journey. Patients who have a mild disorder or attain nearly full recovery can be discharged directly from the hospital to their home. If impairments are hindering the older adults from an independent life at the end of the acute hospital stay, rehabilitation is indicated. If rehabilitation is entirely successful, the older adult is discharged to the former social and professional setting. In the contrary case, the question arises whether the older adult should continue rehabilitation as an outpatient or should be discharged to home care or to a nursing home. Home care is needed when the older adults, even with the support of the family, cannot regain independence in the activities of daily living. Hospital to home transition is recognized as a critical period in the continuum of patient care, where notably high numbers of adverse events and hospital readmissions may oc$\mathrm{cur}^{5,6}$. Among other interventions, application of innovative eHealth strategies as in REACH could mitigate the determinants of these adverse events.

These insights call for a close cooperation of all use cases (hospital, rehabilitation hospital, nursing, and social service for home care and nursing homes). The REACH system aims to establish a close collaboration in a network of partners with experience in smart sensing, monitoring and intervention tech-

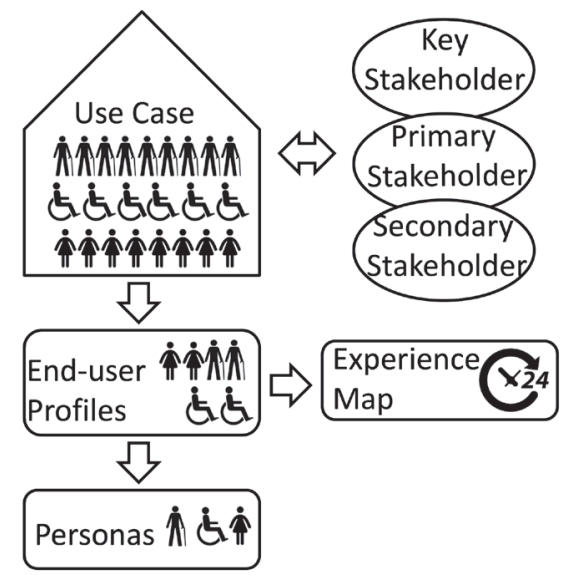

Figure 1. Possible connections between the use cases in $\mathrm{REACH} \mathrm{H}^{12}$ nologies, and industrial partners. Advanced and complex technology platforms will be created for more structured environments such as hospitals or rehabilitation centers. To support the older adults in home care and smart home contexts, an adapted and simplified form is also needed.

In developing a complex user-centered system such as the REACH system, it is essential to perform a requirement specification at the beginning of the project that includes a description of the end-user of the system. The description was substantiated by creating personas-fictional characters representing a typical user of the REACH system-for each use case ${ }^{7}$. The REACH system is not related only to the older adults themselves. It also affects or can be affected by individuals, groups, or institutions that are related to the targeted enduser. These stakeholders need to be identified and analyzed to provide an overview of constraints, incentive structures, and interdependencies ${ }^{8,9}$.

This paper gives an overview of the methods used to identify and analyze stakeholders, personas, and experience maps. Considering the multi-disciplinarity of the project partners, the researchers have to work with different levels and sets of knowledge regarding the use case settings. To provide a common basis and allow a deep insight into the specific processes, we generated personas (based on end-user profiles), experience maps, stakeholder specifications, and analyses for each use case (Figure 2).

\section{Methods}

In the REACH system, the use cases were used to identify, clarify, and organize system requirements. The use cases were defined during the application phase by including different professional caregivers into the consortium. The 4 use cases cover the care continuum of the patients/ older adults and frame the system development process (Figure 1). For each of the 4 use cases, the following methods were applied to identify the requirements for the REACH system.

\section{Stakeholder definition and analysis}

The purpose of stakeholder definition and analysis is to indicate whose interests should be taken into account when making a decision and to indicate why those interests should be considered ${ }^{10}$.

Identifying stakeholders is usually an iterative process, during which additional stakeholders are added as the analysis continues: for example, using expert opinion, focus groups, semistructured interviews, snowball sampling, or a combination of these ${ }^{11}$. The method focuses on 2 key elements: groups or actors are analyzed in terms of: (1) the interest they take in a particular issue and, (2) the quantity and types of resources 


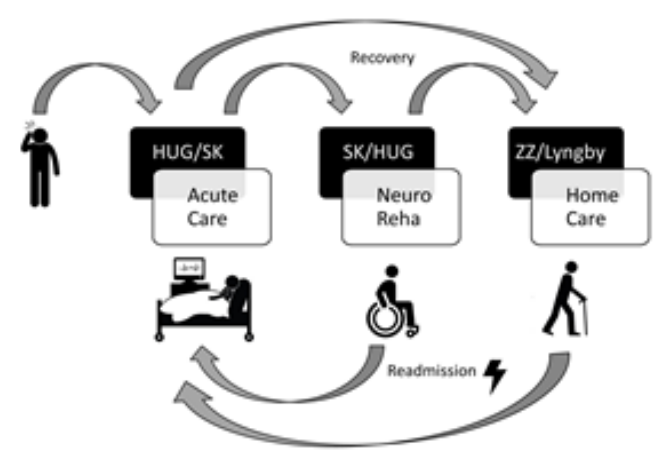

Figure 2. Connection between the use cases, personas, experience maps and stakeholders

they can mobilize to affect outcomes regarding that issue. The term stakeholder analysis encompasses a range of different methodologies for analyzing stakeholder interests and is not a single tool ${ }^{10}$. The stakeholder definition was performed by collecting all identifiable stakeholders into an actor's map. An actor's map is a commonly used method in service design ${ }^{12}$. It represents the system of actors (stakeholders) and their mutual relationships to provide a systematic view of the service and its context. An actor's map can be generated based on the observation of the service from a specific point of view that becomes the center of the representation. If the selected perspective is centered on the end-user (patients and the older adults), the graph will show all of the stakeholders starting from their relationship with him. To identify the stakeholder, processes and contacts were examined where the potential end-user was directly involved. In the second step, all direct contact partners of the potential end-user (natural persons and institutions) were interviewed to identify secondary stakeholders, for example, insurance companies, governmental authorities, and employers. Use case internal workshops were also performed to include all specialists in the analytic process. This method is recommended as highly efficient when more than 3 users work on the stakeholder analysis ${ }^{10}$.

In the 4 REACH use cases, stakeholder specification and analysis was conducted to understand the similarities and differences among the 4 sites by utilizing shared formats. This supports shared understanding among consortium members considering constraints, incentive structures, and interdependencies among stakeholders, and thus the space in which the REACH solution should fulfill unmet needs of the users, both rationalsomatic and emotional-social. The stakeholder analysis should not be expected to provide a future-scenario analysis (for example, how the REACH system would work in practice 5-10 years in the future). But it can provide a necessary overview of the forces-pulls and pushes, incentives, and drives as well as concerns and risks - that will influence the adoption and use of the REACH system and its associated services.

The analysis differentiates between primary, secondary, and key stakeholders. Primary stakeholders are directly influenced by an action, that is, an intervention, a socio-technical design, or a service. Secondary stakeholders are indirectly affected by the action. Key stakeholders do not belong to the former 2 groups but have significant influence on the action, for example, the use of the socio-technical design. The REACH system aims at 65+ seniors, so it is important to identify and characterize their relationships among those who may have power to aid and persuade the older adults (for example, family, friends, and caregivers) and who may have an interest in care and assistive technologies.

To ensure maximal benefits from a stakeholder analysis while keeping the scope at a manageable and practical level, we selected 3 stakeholder templates (Figure 3). They are (1) stakeholder list ${ }^{13,14}$, which is a table with stakeholder characteristics; (2) the onion diagram ${ }^{15}$ and (c) the stakeholder matrix ${ }^{16,17}$. The stakeholder list identifies details of the directly involved stakeholders as well as the most important key ones, with the following 8 aspects: roles, interests, knowledge, expectations, influence, tangible incentives, intangible incentives, and risks. The aspects are meant to capture not only the relatively obvious characteristics but also the more implicit ones. The onion diagram, which arranges layers of the business system, organization, and environment, identifies the relative importance among key stakeholders by setting our target end-user, the senior $65+$, in the middle of the diagram. The stakeholder matrix is shaped in a 2 by 2 matrix, where the $x$-axis indicates interests or involvements and the $y$-axis indicates influence or power. The matrix represents stakeholders' relative power and interest in the use of the technology. For example, the second quadrant is the 'promoters' space; stakeholders allocated to this quadrant should be managed closely in decision making and their ideas should be noted. Each of the local REACH use cases worked independently with the templates. Therefore, it was fundamental that they were simple and easy to use, even for analysts who are not familiar with stakeholder analysis, and still sufficiently powerful (expressive and informative) to provide informative results.

\section{Personas}

The functionalities of the REACH system should accommodate end-users' needs. In hospital settings most of the information is generated through a multitude of caregivers. Consequently, to develop a suitable system, it is crucial to 


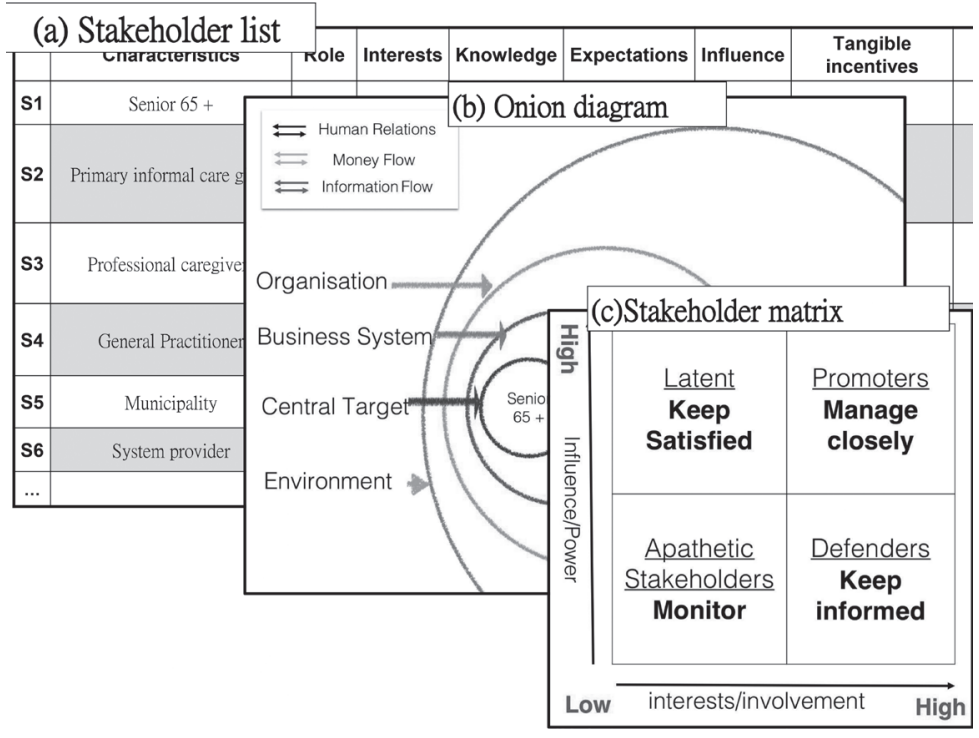

Figure 3. Three templates for stakeholders identification ${ }^{27}$ tion. They can help to better understand customers. As a result, personas are fictional, generalized characters representing the real and potential customers with various needs, objectives, and behavior patterns. The special aspect of a persona description is to focus on the domain you are working within, to highlight the relevant attitudes, and to describe the specific context associated with the area of work ${ }^{19}$.

Nielsen offers 4 different perspectives when generating personas: Alan Cooper's goal-directed perspective; Jonathan Grudin, John Pruitt, and Tamara Adlin's role-based perspective ${ }^{20}$; Sønderstrup-Andersen's

collect, evaluate, and aggregate relevant data. The best way to visualize the needs of end-user groups is by creating models based on the data of various patients or the older adults with different deficits, that is, creating end-user profiles (EPs). SK, for example, concentrated on 2 major deficit categories, motor deficits and cognitive or speech deficits. Five EPs were specified to create 2 personas, 1 with a focus on motor deficits and 1 with a focus on cognitive deficits. SK in addition screened the data from 10 potential end-users with comparable pathologies to ensure that every aspect regarding the pathologies is covered by the personas.

Data on the following items were collected and analyzed: demographics, diagnosis, relevant complications, neuropsychological limitations, and movement disorders.

To follow the REACH objectives, the needs of each persona were structured and assigned to respective phases of the day (Table 1).

Patients' demographic and clinical characteristics were combined with caregivers' experiences and expectations and integrated into the phases of the day model. Data not available from the hospital information system were collected in workshops and interviews.

Personas were created based on these EPs. The purpose of personas ${ }^{7,18}$ is to represent reliable and realistic characteristics of the target users. These representations should be based on qualitative and quantitative data gathered from real people with characteristics similar to the target populaspective ${ }^{19}$. The first 3 perspectives agree that the persona descriptions should be founded on data. The fiction-based perspective creates personas from the researchers' intuition and assumptions.

The core of the goal-directed perspective is the hypothetical archetype that is described as a unique character with specific details. A larger number of initial personas are condensed into final personas, 1 for each kind of user. This persona is defined by its personal, practical, and company-oriented goals, as well as by the relationship with the product to be designed, the emotions of the persona when using the product, and the goals of the persona in using the product. These perspectives provide a focused design and support target-oriented solutions. Every project has its own set of personas.

The role-based perspective also focuses on behavior, but furthermore incorporates data from quantitative and qualitative sources. There should be a clear relationship between the initial data and the persona description. This method should be used in tandem with other methods and provide additional data, for example: market influence, experience of a typical day, and strategic and tactical considerations. These kinds of personas are interchangeable between different projects.

The engaging perspective is rooted in the ability of stories to produce involvement and insight. Through an understanding of characters and stories, it is possible to create a vivid and realistic description of fictitious people, looking at the whole person. The purpose of this perspec- engaging perspective; and the fiction-based per- 
Table 1. Phases of the day in a rehabilitation hospital

\begin{tabular}{ll}
\hline $\begin{array}{l}\text { Phases of the day/ } \\
\text { Quality of life aspects }\end{array}$ & Potential support intensive actions \\
\hline Sleep & $\begin{array}{l}\text { Sleep duration and quality/ monitoring } \\
\text { Bedding during nighttime (position) }\end{array}$ \\
\hline Getting up/ day to night transition & Transfer out of/ into bed \\
\hline Nutrition, fluid intake, dysphagia & $\begin{array}{l}\text { Preparation of meals } \\
\text { Support when eating }\end{array}$ \\
\hline Personal hygiene & Toilet use \\
& Teeth brushing \\
& Showering \\
\hline Dressing & Appropriate clothing \\
& Challenging clothes (e.g., shoes, buttoned shirts, \\
& Sock) \\
\hline Activities (therapies) & Physical therapy \\
& Occupational therapy \\
& Swallowing/ speech therapy \\
\hline Health- conductive behavior & Health(y) activities \\
& Monitoring of health status \\
\hline Autonomy/ functional & Indoor (range of motion in patient room/ at ward) \\
& Outdoor (range of motion in hospital/ hospital area) \\
\hline Social interaction/ participation & Visits from/ contact with/ interaction with \\
& Family/ close friends \\
& Caregiver/ Fellow patients \\
\hline
\end{tabular}

groups that represent your target users. Forth, refine by combining and prioritizing the rough personas. Separate them into primary, secondary, and, if necessary, complementary categories. Finally, make them realistic by developing the appropriate descriptions of each personas' background, motivations, and expectations. Do not include a lot of personal information. Personas generally include the following key pieces of informa-

tive is to enable the designers to actively involve themselves in the lives of the personas instead of focusing on behavior related to the product or the development process. The development should be based on a broad knowledge of the users, combined with data about the social backgrounds, their psychological characteristics, and their emotional relationship with the focus area. The persona descriptions balance data and knowledge about real applications and fictitious information that is intended to evoke empathy. This way, the persona method is a defense against automated thinking.

Fiction-based personas are based on the project teams' assumed understanding of their potential users. They provide a starting point from which the developer can begin evaluating products and come up with early design hypotheses. Fictionbased personas are used to create an empathetic focus on the design process and help designers to be reflexive when creating scenarios. The validity and value of these methods is questionable due to the sole use of fictitious elements.

According to the U.S. Department of Health and Human Services ${ }^{15}$, there are a few important steps when creating personas. First, a user specification needs to be conducted by answering the following questions: Who are your users and why are they using your products and services? What are their behaviors, assumptions, limitations, and expectations? Second, the specification should be condensed by looking for characteristics that are specific, relevant, and universal to your products and services and their users. Third, brainstorm by organizing elements into persona tion: fictional name, job titles and major responsibilities, demographics such as age, education, ethnicity, and family status, the related goals and tasks they are trying to complete, their physical, social, and technological environment, a quote that sums up what matters most to the persona as it relates to relevant products and services, and casual pictures representing that user group. It is important to organize persona information in an easy to read, logical format.

SK generated 2 personas based on the data set of 5 patients who represented the following neurological pathologies: stroke (single and multiple), Alzheimer's disease, Parkinson's disease, and critical illness polyneuropathy. One male persona represents patients with severe motor deficits and minor cognitive deficits, and one female persona (Antonia) represents patients with severe cognitive deficits and minor motor deficits. In the results section, Antonia is described in detail as an example of information that could be included in a persona and in which form a persona could be presented (Figure 4).

Based on data from clinical routine assessments (for example, the Mini-Mental Assessment or the Barthel Index) the cognitive, psychological, and mobility profile of the persona was generated. The most common socio-demographic and medical characteristics from the source patients were used to describe the social environment and medical profile of Antonia. Physicians, therapists, and nursing staff were interviewed to evaluate typical therapy goals and problems of patients with severe cognitive and minor motor deficits. 


\section{Design requirements for active aging}

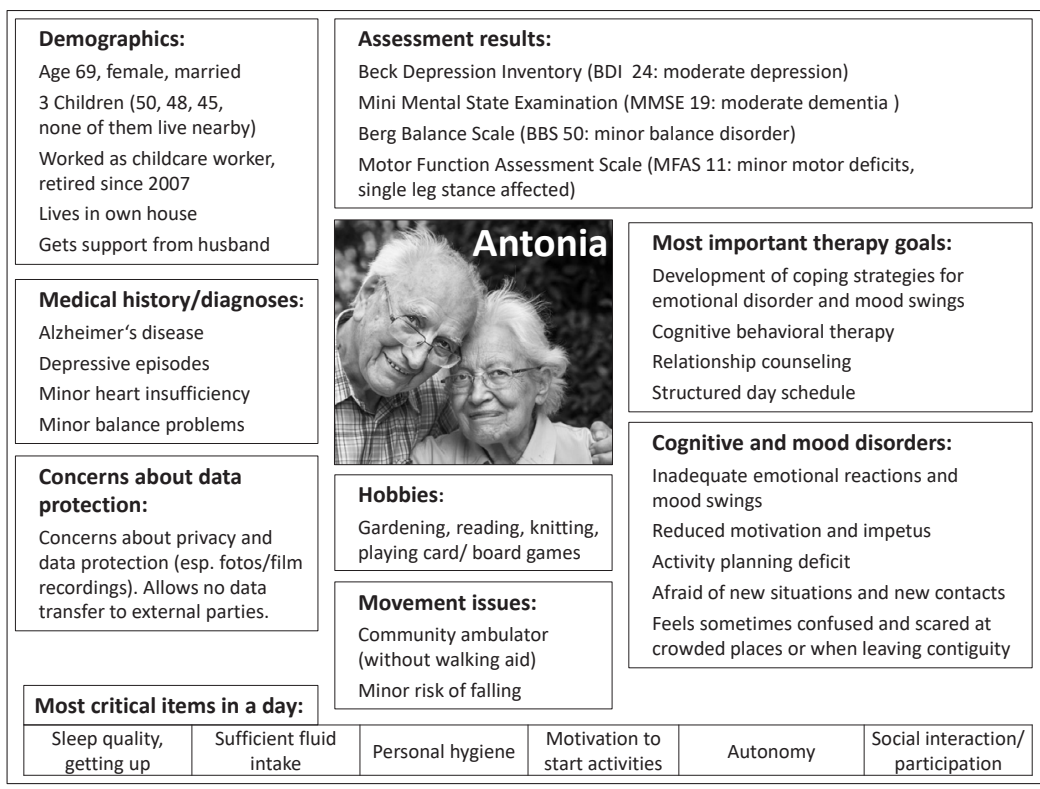

Figure 4. Persona template for Antonia ${ }^{12}$ using online ethnography, observing people, and shadowing them as they go through a typical day. The team should keep an open mind at every stage of the process. When doing fieldwork, it is best to refrain from thinking and talking about the desired solution or direction. Instead, aim for talking about the experiences and the issues in their context. Using the current and real experiences captured during fieldwork, the team starts compiling an EF poster and issue cards. The user needs, mental status, emotions, activities, and interaction phases

\section{Experience mapping}

The experience map (EM) visualizes situations, actions, emotions, and contacts that a use case resident may experience during a typical day. The method and the description in this section are partly adopted from "Experience Flows: Understanding People and their Experiences to Deliver Meaningful Innovations" ${ }^{\prime 21}$.

EMs are one of Philips' most useful tools for creating people-centered solutions. They help to spot and contextualize the unmet needs of people and then translate these into innovation opportunities and directions. This is done by consolidating vast amounts of qualitative and quantitative information into a graphical representation that makes immediate sense to everyone. Using multiple perspectives on a particular issue or topic ensures that a holistic insight into the total user experience is created ${ }^{22}$.

As a way of mapping an experience from expectation to first impression then through discovery, usage, and finally to memory, Philips developed the experience flow (EF). In addition to providing detailed insights from an older adult/patient perspective, the process of creating a flow also helps a team to adopt and understand multiple perspectives and approaches to a context. Using people-centered research, the team works with the project's target group to uncover older adults'/ patients' thoughts and feelings in a specific situation. This can be done in a number of ways, which include formal and informal interviews, workshops with stakeholders, asking individuals to write down their experiences in a diary or by are collected in this material. This helps to visually pinpoint problems or gaps and serves as a basis for identifying opportunities across the EF in a collaborative workshop. The team uses the poster to walk through the journey as if they were the person or people concerned. Then they discuss, challenge, and enrich the journey by spotting areas where the person's needs are not being met.

As a final step, similarities and differences were summarized with respect to 3 aspects: design (graphic visualization), content (elements included into the experience map), and complexity (simple: one user, one goal, one scenario, one path, or a multidimensional experience map).

\section{Results}

\section{Stakeholder definition and analysis}

Stakeholders and their characteristics and relationships to the 4 use cases were described and analyzed utilizing the aforementioned templates (Figure 3). Experienced therapists and scientists performed the task of generating the stakeholder analysis of each use case with in-depth knowledge of the end-users, the stakeholders, and the use case characteristics. They received the 3 templates and a self-help instruction document to conduct the stakeholder analysis.

LYNGBY analyzed 6 stakeholders. Since caretaking is carried out in home settings, the core stakeholders are informal and formal caregivers, municipalities, and public authorities. Medical personnel beyond municipal nurses are not included among the stakeholders, and the medical services and treatment at the hospitals are out of scope. 
ZZ analyzed 7 stakeholders, among which informal stakeholders such as primary informal caregivers (relatives and friends) and the Meet and Greet Centre's community hold the biggest and most important roles. Insurance companies and municipalities are at present outside of the circle of care, but are expected to play an important role in the near future.

HUG analyzed 6 stakeholders. The informal supporters, such as caregivers and hospital caregivers, have a great influence on the patients despite the fact that the medical caregiving system considers informal caregivers to be less crucial. Insurance companies are also identified as key stakeholders.

SK analyzed 10 stakeholders, the biggest number among the 4 locations. This also indicates that there is a complex support system both in formal as well as informal relation to the patient at the rehabilitation hospital.

One major difference across the 4 use cases is their use context. One use context is the hospital (SK and HUG) where professional caregivers are constantly available and where the patients are typically monitored several times a day. The other use context is the population of older adults living at home, with daily or weekly assistance. For this latter user group, professional caregivers (nurses and nurse assistants) are available only for scheduled visits and emergencies. These conditions lead to differences in societal characteristics and the care continuum.
Differences in societal characteristics are obvious, for example, regarding the role of insurance. In a home care setting such as ZZ and LYNGBY, insurance companies play a less critical role compared to HUG and SK. As social-welfare country, Denmark has considerable senior care within the national care package for social and health needs, covered entirely by taxes. Insurance companies thus play a negligible role, covering dentistry, hearing aids, and a small part of medication. For ZZ, the role of insurance companies is different, since the Netherlands has a dual-level system ${ }^{23}$. All primary and curative care (the family doctor service and hospitals) is financed from mandatory private insurance. Long-term care for the older adults, terminally ill, long-term mentally ill, etc., is covered by social insurance. For HUG and SK, where insurance companies have a key role, it is more important to consider insured budgets for prevention and treatment. Due to the societal differences, the influence of stakeholders differs.

Care continuum is another key factor. HUG and SK are, as mentioned above, hospitals where the older adults are formally patients, and where more formal medical treatments and treatment-related exercises are involved. LYNGBY and ZZ settings are caregiving and daily care settings, where daily or weekly support and active living are central. According to the treatment stage in the continuum of care, influence and roles of stakeholders differ.

Both informal and formal support is important in the health care setting for seniors. Differences in the setting as well as socio-political factors and the treatment stage influence the level of importance of informal and formal support along the treatment process. Informal caregivers usually have a strong impact on the daily life of seniors while sometimes in the hospital use cases have less influence on the formal treatment process. In this setting, the informal caregivers' impact on the REACH system could also be low.

Figure 5. Onion diagram for $\mathrm{HUG}^{27}$ 


\begin{tabular}{|c|c|c|c|c|}
\hline \multicolumn{5}{|c|}{ Experience map - one morning of a patient in neurological rehabilitation } \\
\hline Timeline & 6 & 10 & 11 & 12...continued \\
\hline Experience & $\begin{array}{l}\text { Gets woken up, vital parameter } \\
\text { check, bed to wheel chair, } \\
\text { showering/toileting, medication } \\
\text { intake, breakfast (via PEG) }\end{array}$ & $\begin{array}{l}\text { Therapies: physical/ dysphagia/ NP } \\
\rightarrow \text { practices swallowing, UE } \\
\text { function, structure planning } \\
\text { (group therapy) }\end{array}$ & Physician's visit & $\begin{array}{l}\text { Lunch (PEG), } \\
\text { medication } \\
\text { intake }\end{array}$ \\
\hline Location & Patient room/ bathroom & Therapy rooms & Physician's office & Patient room \\
\hline Equipment & $\begin{array}{l}\text { Bed/ wheel chair, shower chair } \\
\text { feeding tube/ enteral nutrition }\end{array}$ & $\begin{array}{l}\text { Amadeo robot, } \\
\text { dysphagia nutrition }\end{array}$ & Wheel chair & Feeding tube \\
\hline Contacts & Nurse & Therapists & Physician/ Assistant & Nurse \\
\hline Mood & $\Theta \bigodot$ & & (3) & \\
\hline Condition & Tired/motivated & Alert/frustrated & Delighted & Tired/depressed \\
\hline $\begin{array}{l}\text { Abbreviations: } \\
\text { NP - Neuropsyc } \\
\text { PEG - Percutane } \\
\text { endoscopic gast } \\
\text { UE - Upper extr }\end{array}$ & $\begin{array}{l}\text { Would prefer to sleep longer. } \\
\text { Feels good about doing teeth } \\
\text { brushing, and combing on his } \\
\text { own; needs only minor help } \\
\text { for dressing. }\end{array}$ & $\begin{array}{l}\text { Enjoys food in swallowing therapy, but } \\
\text { chokes two times. No problems with } \\
\text { structure planning tasks in NP group } \\
\text { therapy. Starts motivated with arm } \\
\text { training but gets angry due to mistakes } \\
\text { in fine motor deficit exercises. Amadeo } \\
\text { robot automatically adapts to lower } \\
\text { level. Therapist tries to motivate } \\
\text { patient to work on adapted level but } \\
\text { patient feels frustrated nontheless. }\end{array}$ & $\begin{array}{l}\text { Physician discusses the } \\
\text { progress and suggests } \\
\text { discharge next week. } \\
\text { Explains further } \\
\text { procedures (e.g., } \\
\text { therapies after } \\
\text { discharge, mobile } \\
\text { nursing service) and } \\
\text { informs about } \\
\text { available medical aids. }\end{array}$ & $\begin{array}{l}\text { Is depressed } \\
\text { because he wants } \\
\text { to go back to oral } \\
\text { food intake. Feels } \\
\text { tired and takes a } \\
\text { nap. Afterwards } \\
\text { his mood has } \\
\text { slightly improved. }\end{array}$ \\
\hline
\end{tabular}

Figure 6. Experience map in rehabilitation hospita/ ${ }^{12}$

The onion diagram of HUG is an example (Figure 5). The senior $65+$ is allocated in the center, and other stakeholders such as home caregivers, hospital caregivers, technicians, and insurance companies are allocated as equally important business partners with different relational strengths. In the diagram, informal caregivers (the most outer stakeholder in Figure 5) are allocated outside of the formal treatment process.

\section{Personas}

In the following, the female persona with minor motor and major cognitive deficits is described. The graphical representation is simple and easy to read. The information about characteristics is grouped around a centered photo of a typical representative.

Antonia is a married woman with 3 children who live too far away to support her in her daily life but are available for special occasions, e.g. renovating the house and organizing family celebrations. For ADL, she is dependent on the support of her husband, who finds it challenging to motivate Antonia and initiate activities with an adequate level of stimulation. Her mental health problems and the intermittent depressive episodes impede her autonomy and social participation. Her husband is responsible for generating a structured environment to fulfill her increasing need for safety. Lack of sleep due to Antonia's sleeping disorder, a common symptom of Alzheimer's disease ${ }^{24}$, frustration about her decreas- ing ability in the basic activities of daily living, her inadequate reactions and mood swings, and the loss of spontaneity sometimes exhaust him. This leads to impatient and angry behavior that has to be addressed in relationship counseling.

REACH could possibly support Antonia to perform cognitive training, guide her through household and other routine activities, recommend sufficient new activities, provide information to address her safety needs, and remind her to drink enough. This would enhance her autonomy, reduce the support needed from her husband, and therefore increase the quality of life for both.

\section{Experience mapping}

REACH's activities to collect and map the experiences and opportunities are abstracted from a more extensive set of guidelines on experience flows ${ }^{21}$. The procedure described in the following covers the most important aspects: Frame the project by clearly defining the parameters, for example, target persons, regions, challenges, timing, core teams, and deliverables. (Figure 6) shows a schematic example for a typical day in a rehabilitation setting. The timeline is set on the horizontal axis, and the vertical axis shows the different layers of insight.

For the application in REACH, we used different layers (Figure 6). Mood and experience both describe how the older adult/patient feels 
about specific events, activities, and interactions throughout the day. Equipment indicates the technology used. This can be rehabilitation equipment and technology to communicate with their family. Social contacts describe the interactions with friends, family, and others who are an important part of the patient's life. Depending on the context, personality, and available technology, people may engage in different types of social contacts and activities and experience them in different ways. Medical professionals and stakeholders indicate which medical professionals and institutions are involved at certain points during the day.

\section{Discussion, Conclusions, and Limitations}

The results described indicate the requirement to design a highly modular concept. This conclusion is based on the finding that the user population is heterogeneous regarding health and mental status and kind of additional support available. Furthermore, REACH has to be malleable to fit into the different user environments in an unobtrusive manner. The development should follow the rules of the interior design concept to achieve high user acceptance ${ }^{25}$. The modularity must also be able to follow changes in user needs, for example, those caused by health improvement or deterioration. Extensive consultation with specialized field experts (hygiene officers, therapists, psychiatrists, and functional designers) and potential users assures the implementation of detailed requirements from the stakeholders. The stakeholders should be involved in the development of the preliminary idea, the reviewed and revised concept, and the final result. The client approval for the final concept will be secured by constant review and revision after every development and consultation step ${ }^{22}$.

After the development process, extensive and individualized user training will be necessary to enhance technical acceptance. The users should also be informed about the modular structure and the options resulting from the modularity ${ }^{26}$.

The stakeholder analysis of the four use cases provided interesting insights that indicate further benefits of the REACH system and subsystems developed for future care settings. Some stakeholders also expressed concerns that must be taken into account to gain a high user acceptance of the REACH system.

For the primary users (the older adults), the main risks and drawbacks are their fear of data disclosure and the stress associated with the use of technology. These risks are similar for relatives and friends (informal caregivers) and, to some extent, for the formal caregivers as well. For the latter group (municipality), there is the concern of being responsible for inadvertently disclosing data as well as the burden on budgets for deploying and maintaining a system such as REACH. Insurers have the additional concern that they may be accused of misusing data.

The benefits for the primary users and their families and friends are the greater autonomy that the system may provide, more independence, and extra self-determination. Relatives expect that the REACH system will simplify the organization and supporting activities. For professional caregivers, improved efficiency in care (including better understanding of patient needs), more information about patients' activities or habits between treatments and visits, and early warning and easier monitoring of changes in health status is more relevant. In addition to the aspects already mentioned, the REACH system should also support a smoother transition from rehabilitation hospital to home, when relevant. Finally, for insurers and funding entities, the benefits rely on the prospect of getting an economically sound use of resources, lower costs due to the prevention of readmission, hospitalization, and transition to (costly) long-term care.

The similarity of the results indicates the shared challenges and potential across different use cases. Despite similarities in the medical and therapeutic approaches, there are major differences in the healthcare ecosystems in different countries. Thus, comprehensive analysis has to include both the user-centered approach with the use cases from hospital to home care as well as the healthcare value chain perspective from the business model approach. Considering the target user (65+ seniors), the future REACH system and subsystems and the REACH business model will have to take into account the care level of the target user as well as societal medical settings.

All descriptions and analyses in this project were initially based on the use case concept. The use cases were located in four northern European countries, which is not representative of the whole European or global market. The use case concept was limited to the determination of requirements. A cost-benefit trade-off analysis was not performed in order to allow an unrestricted development process. It is possible that a selection bias was generated due to a restricted number of end-user profiles. Those who support the evaluation process may have a higher acceptance of technical support systems than the average population. 


\section{Acknowledgments}

We would like to thank the contributors to reports 1.1 and 1.2 for their exceptionally positive and productive collaboration: Technical Universities Munich, Eindhoven, and Denmark, University of Copenhagen, Ecole Polytechnique Lausanne, Philips International, Zuidzorg, Lyngby, Hopitaux Universitaires de Geneve, Alreh Medical.

The project has received funding from the European Union's Horizon 2020 research and innovation programme under grant agreement No 690425.

\section{References}

1. Jacobson I. Use cases-Yesterday, today, and tomorrow. Software and Systems Modeling 2004;3(3):210-220; https://doi.org/10.1007/ s10270-004-0060-3

2. Ministry of Social Welfare-Ministry of Health and Prevention. National Report on Strategies for Social Protection and Social Inclusion. Denmark, European Commission, Talinn 2008-2010

3. Schulz E. The long-term care system for the elderly in Denmark 2010. ENEPRI Research Report No. 73. Contribution to WP1 of the ANCIEN Project. ISBN 978-94-6138-015-9; http://www. ancien-longtermcare.eu/sites/default/files/ENEPRI\%20_ANCIEN_\%20RRNo.73DenmarkREV2. pdf; retrieved October, 2, 2017

4. Vermeulen J, Neyens JC, van Rossum E, Spreeuwenberg MD, de Witte LP. Predicting ADL disability in community-dwelling elderly people using physical frailty indicators: a systematic review. BMC geriatrics 2011;11(1):33; https://doi. org/10.1186/1471-2318-11-33

5. Baker DW, Einstadter D, Husak SS, Cebul RD. Trends in postdischarge mortality and readmissions: Has length of stay declined too far? Archives of internal medicine 2004;164(5):538-44. Epub 2004/03/10; https://doi.org/10.1001\%2Farc hinte.164.5.538

6. Forster AJ, Murff HJ, Peterson JF, Gandhi TK, Bates DW. The incidence and severity of adverse events affecting patients after discharge from the hospital. Annals of internal medicine 2003;138(3):161167; https://doi.org/10.7326/0003-4819-138-3200302040-00007

7. Pruitt J, Grudin J, editors. Personas: practice and theory. In: Proceedings of the 2003 conference on Designing for user experiences. ACM Press 2003; https://doi.org/10.1145\%2F997078.997089

8. Bourne L. Making projects work: Effective stakeholder and communication management: CRC Press Auerbach Publications 2015;pp 1-22; https:// doi.org/10.1201\%2Fb18100-2

9. Alexander I. A taxonomy of stakeholders, human roles in system development. In: Issues and Trends in Technology and Human Interaction. UK: Scenario Plus Ltd. \& City University 2006; pp 25-71; https://doi.org/10.4018\%2F9781599042688.ch002

10. Crosby B. Stakeholder analysis: a vital tool for strategic managers. USAID's Implementing Policy Change Project, Technical Notes; 1991; http://www.msiworldwide.com/wp-content/uploads/2011/07/IPC_Stakeholder_Analysis_A_Vi-
tal_Tool_for_Strategic_Managers.pdf; retrieved October 2017

11. Reed MS, Graves A, Dandy N, Posthumus H, Hubacek K, Morris J, Prell C, Quinn CH, Stringer LC. Who's in and why? A typology of stakeholder analysis methods for natural resource management. Journal of environmental management 2009;90(5):1933-1949; https://doi.org/10.1016/j. jenvman.2009.01.001

12. Deliverable D1 "Use Case Analysis"-Report (Public) M3; http://reach2020.eu/?page_id=1190; retrieved August 2017

13. Hosseini JC, Brenner SN. The stakeholder theory of the firm: A Methodology to Generate Value Matrix Weights. Arnold DG, editor. Business Ethics Quarterly. UK: Cambridge University Press 1992;2(2):99-119; https://doi. org/10.2307/3857566

14. Schmeer K. Stakeholder analysis guidelines. Policy toolkit for strengthening health sector reform (section 2). Latin America and Caribbean Regional Health Sector Reform Initiative 1999; pp 1-43; http://www.who.int/workforcealliance/knowledge/ toolkit/33.pdf; retrieved September 13, 2017

15. US Department of Health and Human Services: Personas; https://www.usability.gov/how-to-and-tools/ methods/personas.html; retrieved April 20, 2016

16. Polonsky MJ. Stakeholder management and the stakeholder matrix: potential strategic marketing tools. Journal of Market-Focused Management 1996;1(3):209-29; https://doi.org/10.1007/ bf00190039

17. Mitchell RK, Agle BR, Wood DJ. Toward a theory of stakeholder identification and salience: Defining the principle of who and what really counts. Academy of management review 1997;22(4):853-886; https://doi.org/10.5465/amr.1997.9711022105

18. Matthews T, Judge T, Whittaker S. How do designers and user experience professionals actually perceive and use personas? In Proceedings of the SIGCHI conference on human factors in computing systems. ACM 2012; pp 1219-1228; https://doi. org/10.1145/2207676.2208573

19. Nielsen L. Personas. In: Soegaard M and Dam RF (editors.). The Encyclopedia of Human-Computer Interaction, 2nd Ed. Aarhus, Denmark: The Interaction Design Foundation 2013. ISBN-10: 1591405629. ISBN-13: 978-1591405627; http:// www.interaction-design.org/encyclopedia/personas.html; retrieved September 13, 2017

20. AIGA: Adlin T, Pruitt J. Why is it so Hard to Make Products that People Love? 2006; http://www. aiga.org/why-is-it-so-hard-to-make-products-thatpeople-love/; retrieved August 3, 2017

21. Philips Design \& Innovation Communications KPNV: Experience Flows Understanding people and their experiences to deliver meaningful innovations; http:// www.newscenter.philips.com/pwc_nc/main/design/ resources/pdf/Inside-Innovation-Backgrounder-Experience-Flows.pdf; retrieved May 30, 2017

22. Pickles J, Hide E, Maher L. Experience based design: a practical method of working with patients to redesign services. Clinical Governance: An International Journal 2008,13(1):51-58; https://doi. 


\section{Design requirements for active aging}

org/10.1108/14777270810850634

23. Government of the Netherlands: Topic: Health Insurance; https:/www.government.nl/topics/ health-insurance; retrieved May 29, 2017

24. Bliwise DL. Sleep disorders in Alzheimer's disease and other dementias. Clinical cornerstone 2004; https://doi.org/10.1016/S1098-3597(04)90014-2

25. Haddad R. Research and Methodology for Interior Designers 2013. 2nd World Conference on Design, Arts and Education DAE. Procedia-Social and Be- havioral Sciences 122; 2014; pp 283-291; https:// doi.org/10.1016/j.sbspro.2014.01.1343

26. Venkatesh V, Speier C, Morris MG. User acceptance enablers in individual decision making about technology: Toward an integrated model. Decision sciences 2002;33(2):297-316; https://doi. org/10.1111/j.1540-5915.2002.tb01646.x

27. Deliverable D41 "REACH Stakeholder Management"-Report (Public) M12; http://reach2020.eu /?page_id=1190; retrieved August 07, 2017 